\title{
Interruptin B induces brown adipocyte differentiation and glucose consumption in adipose-derived stem cells
}

\author{
SIREEWAN KAEWSUWAN ${ }^{1,2}$, ANUCHIT PLUBRUKARN ${ }^{1}$, MALEERUK UTSINTONG $^{3}$, SEOK-HO KIM $^{4}$, \\ JIN-HYUN JEONG ${ }^{5}$, JIN GU CHO ${ }^{6}$, SANG GYU PARK ${ }^{6}$ and JONG-HYUK SUNG ${ }^{5}$ \\ ${ }^{1}$ Department of Pharmacognosy and Pharmaceutical Botany; ${ }^{2}$ Excellent Research Laboratory, \\ Phytomedicine and Pharmaceutical Biotechnology Excellence Center, Faculty of Pharmaceutical Sciences, \\ Prince of Songkla University, Songkhla 90110; ${ }^{3}$ School of Pharmaceutical Sciences, University of Phayao, Phayao 56000, \\ Thailand; ${ }^{4}$ Department of Pharmacy, College of Pharmacy, CHA University, Pocheon 487-600; \\ ${ }^{5}$ Department of Pharmaceutics and Institute of Pharmaceutical Science, College of Pharmacy, Yonsei University, \\ Incheon 405-750; ${ }^{6}$ Department of Pharmacy, College of Pharmacy, Ajou University, Suwon 16499, Republic of Korea
}

Received January 31, 2015; Accepted November 17, 2015

DOI: $10.3892 / \mathrm{mmr} .2016 .4758$

\begin{abstract}
Interruptin $B$ has been isolated from Cyclosorus terminans, however, its pharamcological effect has not been fully identified. In the present study, the effects of interruptin $\mathrm{B}$, from $C$. terminans, on brown adipocyte differentiation and glucose uptake in adipose-derived stem cells (ASCs) were investigated. The results revealed that interruptin $\mathrm{B}$ dose-dependently enhanced the adipogenic differentiation of ASCs, with an induction in the mRNA expression levels of peroxisome proliferator-activated receptor (PPAR)- $\alpha$ and PPAR $-\gamma$. In addition, interruptin B efficiently increased the number and the membrane potential of mitochondria and upregulated the mRNA expression levels of uncoupling protein (UCP)-1 and cyclooxygenase (COX)-2, which are all predominantly expressed in brown adipocytes. Interruptin B increased glucose consumption in differentiated ASCs, accompanied by the upregulation in the mRNA expression levels of glucose transporter (GLUT)-1 and GLUT-4. The computational analysis of molecular docking, a luciferase reporter assay and surface plasmon resonance confirmed the marked binding affinity of interruptin B to PPAR- $\alpha$ and PPAR- $\gamma\left(K_{D}\right.$ values of
\end{abstract}

Correspondence to: Professor Jong-Hyuk Sung, Department of Pharmaceutics and Institute of Pharmaceutical Science, College of Pharmacy, Yonsei University, 162-1, Songdo-dong, Yeonsu-gu, Incheon 405-750, Republic of Korea

E-mail: brian99@empal.com

Professor Sang Gyu Park, Department of Pharmacy, College of Pharmacy, Ajou University, 206 Worldcup-ro, Youngtong-gu, Suwon 16499, Republic of Korea

E-mail: sgpark@ajou.ac.kr

Key words: interruptin B, Cyclosorus terminans, adipose-derived stem cell, brown adipocyte, peroxisome proliferator-activated receptors, glucose uptake
5.32 and $0.10 \mu \mathrm{M}$, respectively). To the best of our knowledge, the present study is the first report to show the stimulatory effects of interruptin B on brown adipocyte differentiation and glucose uptake in ASCs, through its role as a dual PPAR- $\alpha$ and PPAR- $\gamma$ ligand. Therefore, interruptin B could be further developed as a therapeutic agent for the treatment of diabetes.

\section{Introduction}

Obesity can be defined not only as excess body weight, but also as an increased accumulation of adipose tissue. Obesity is a risk factor for chronic diseases, including hypertension, coronary heart disease, stroke, obstructive sleep apnea, asthma, hyperlipidemia and type 2 diabetes $(1,2)$. Current strategies for obesity management include diet, exercise, drug therapy and bariatric surgery, either alone or in combination. Anti-obesity drugs are designed to suppress appetite and reduce fat absorption $(1,3)$. However, these drugs show adverse effects, including tachycardia, hypertension, arrhythmias, steatorrhea, fecal urgency and incontinence (4). Due to these undesirable effects of anti-obesity drugs, particularly the life-threatening safety concerns of the centrally acting agents, there is an urgent requirement to identify effective, safe and well-tolerated anti-obesity drugs. One strategy for weight loss is to induce brown adipocyte tissue in adult humans. As brown adipocyte tissue generates heat from fatty acids and increases the breakdown of fat in the body, brown adipocyte tissue is considered a promising target to combat obesity (5).

Diabetes mellitus is often associated with obesity, and its incidence is increasing (6). Diabetes results from a defect of insulin secretion and/or insulin action, and thaiazolidinediones (TZDs), including rosiglitazone and pioglitazone, are a class of anti-diabetic drugs, which work as insulin sensitizers $(7,8)$. They usually promote adipocyte differentiation and encourage insulin sensitivity by stimulating the transcriptional activity of peroxisome proliferator-activated receptor (PPAR)- $\gamma(9,10)$. However, its side effects include weight gain, edema with worsening of cardiac failure, liver toxicity and anemia (11-13), 
which has resulted in the withdrawal of certain TZDs from the market. Therefore, there is a requirement to develop novel anti-diabetic drugs.

The adipocyte is a central site for the regulation of energy storage and metabolism (14). Adipocyte differentiation is triggered by signaling molecules, which induce the conversion of adipose-derived stem cells (ASCs) to pre-adipocytes, which finally differentiate into adipocytes. Following a library screen in a preliminary study, a novel regulator of ASC differentiation and glucose uptake was identified from the fern Cyclosorus terminans. Although its traditional use has not been described, this plant is usually consumed as a vegetable in northern Thailand (15). Bioactive interruptins A and B from $C$. terminans, which exhibit antibacterial, anticancer and reactive oxygen species (ROS)-scavenging activities, were identified in our previous study (16). The present study aimed to investigate whether interruptin B isolated from $C$. terminans induces brown adipocyte differentiation of ASCs, whether interruptin B induces glucose uptake by ASCs, and investigate the potential mechanism underlying the action of interruptin B in regulating ASC development. The results from the present study aim to provide a novel scientific basis to support the use of the edible vegetable, $C$.terminans, as a medicinal plant, and to justify applications of interruptin B for the treatment of obesity and diabetes.

\section{Materials and methods}

Materials. Interruptin B $(0.003 \% \mathrm{w} / \mathrm{w})$ from the fern Cyclosorus terminans (J. Sm. ex Hook.) Panigrahi, of the family Thelypteridaceae (Fig. 1A) was purified by open column chromatography over silica gel, using the same step gradient of $n$-hexane with increasing concentrations of dichloromethane, followed by dichloromethane with increasing concentrations of ethyl acetate, and then ethyl acetate with increasing concentrations of methanol, as described in our previous report (16). The plant was collected from a forested area at the Prince of Songkla University (PSU; Songkhla, Thailand) in April 2010 by Miss. Arpaporn Kaewchoothong and was identified by Professor Thaweesakdi Boonkerd (Chulalongkorn University, Bankok, Thailand). The voucher specimen (identification no. SKP 208 0320 001) is now stored in the herbarium of the Faculty of Pharmaceutical Sciences, PSU. The purity of the isolated interruptin B was determined from nuclear magnetic resonance spectra and high-performance liquid chromatography data, which revealed no significant impurities. Rosiglitazone and bisphenol A diglycidyl ether (BADGE) were purchased from Sigma-Aldrich (St. Louis, MO, USA). GW6471 was purchased from Tocris Bioscience (Ellisville, MO, USA). All other chemicals were of reagent grade and obtained from Sigma-Aldrich, iNtRON Biotechnology (Seoul, South Korea) or Molecular Probes (Thermo Fisher Scientific, Inc., Waltham, MA, USA).

In addition, $\alpha$-minimum essential medium ( $\alpha$-MEM) was purchased from Thermo Fisher Scientific, Inc., and fetal bovine serum (FBS) and penicillin-streptomycin were purchased from Gibco (Thermo Fisher Scientific, Inc.). Differentiation media and supplements were purchased from Lonza (Walkerville, MD, USA).
ASC culture. Human ASCs were isolated via the liposuction of subcutaneous fat, as described previously (17), following the provision of written informed consent, at Bundang CHA Hospital (Seoul, Korea; BD2011-152D) and approval by the ethics committee of CHA University (Pocheon, South Korea). The human ASCs were cultured in $\alpha$-MEM supplemented with $10 \% \mathrm{FBS}$ and $1 \%$ penicillin/streptomycin at $37^{\circ} \mathrm{C}$ under a humidified $5 \% \mathrm{CO}_{2}$ atmosphere.

Adipocyte differentiation. For adipocyte differentiation, the ASCs were plated in 6-well plates $\left(4 \times 10^{4}\right)$ and grown in $\alpha$-MEM containing $10 \%$ FBS for 3 days, following which the medium was replaced with pre-differentiation medium (pre-adipocyte growth medium-2; PGM-2) comprising pre-adipocyte basal medium-2 supplemented with FBS, L-glutamine and GA-1000. The cells were further cultured for 6-7 days until reaching confluence. In the subsequent adipogenesis experiments, the ASCs were treated for 4 days with various concentrations of interruptin $\mathrm{B}(0,12.5,25$ or $50 \mu \mathrm{M})$ in pre-adipocyte differentiation medium-2 (PDM-2) comprising PGM-2 supplemented with $h$-insulin, dexamethasone, 3 -isobutyl-1-methylxanthine and indomethacin. Dimethyl sulfoxide (DMSO; 0.1\%) and rosiglitazone $(50 \mu \mathrm{M})$ served as negative and positive controls, respectively.

Oil Red $O$ staining. Following the induction of differentiation of the ASCs for 4 days, the cells were fixed with $10 \%$ formaldehyde (Junsei Chemical Co., Ltd., Toyko, Japan) in phosphate-buffered saline (PBS, iNtRON Biotechnology, Seoul, Korea) for $1 \mathrm{~h}$ at room temperature, washed three times with PBS and stained with filtered Oil Red O (Sigma-Aldrich) solution (0.5\% Oil Red O-isopropyl alcohol: $\mathrm{H}_{2} \mathrm{O} ; 3: 2$, v/v) for $2 \mathrm{~h}$. Following three washes with distilled water, images of the cells were captured under a microscope. Lipid was extracted with isopropanol (Sigma-Aldrich) for $30 \mathrm{~min}$, and the concentration was determined using a microplate reader (Tecan GmbH, Grodig, Austria) at a wavelength of $492 \mathrm{~nm}$.

Glucose consumption assay. The confluent ASCs were treated with interruptin $\mathrm{B}(0,25$ or $50 \mu \mathrm{M})$ and rosiglitazone $(50 \mu \mathrm{M})$ in PDM-2 medium for 4 days. The conditioned medium from the differentiated ASCs was then removed and assayed for glucose content using a Glucose Colorimetric Assay kit II, (cat. no. 686-100, BioVision, Inc., Milpitas, CA, USA).

RNA analysis. The total RNA from the differentiated ASCs treated with varying concentrations of interruptin $\mathrm{B}(0,12.5,25$ or $50 \mu \mathrm{M}$ ) was isolated using an Easy-spin ${ }^{\mathrm{TM}}$ (DNA-free) Total RNA Extraction kit (iNtRON Biotechnology). Subsequently, 500 ng total RNA from each sample was reverse-transcribed to cDNA using 100 ng Oligo(dt) 15 Primer (Promega Corporation, Madison, WI, USA) and a reverse transcription system (Promega Corporation), in accordance with the manufacturer's protocol. Semi-quantitative polymerase chain reaction (PCR) analysis was performed to visualize and compare the expression levels of the genes associated with adipogenesis. PCR was performed using Solg ${ }^{\mathrm{TM}}$ 2X Taq PCR-Pre-Mix (SolGent Co., Ltd., Daejeon, South Korea) in a total volume of $30 \mu \mathrm{l}$ for PCR amplification of cDNA, which was reverse-transcribed from $500 \mathrm{ng}$ total RNA. PCR amplification was performed 
for 35 cycles with denaturation at $94^{\circ} \mathrm{C}$ for $20 \mathrm{sec}$, annealing at $54-58^{\circ} \mathrm{C}$ for $40 \mathrm{sec}$ and polymerization at $72^{\circ} \mathrm{C}$ for $20 \mathrm{sec}$, followed by a final extension at $72^{\circ} \mathrm{C}$ for another $10 \mathrm{~min}$, and cooled to $4^{\circ} \mathrm{C}$. The primer sequences (Bioneer Corporation, Daejeon, South Korea) used for PCR amplification are listed in Table I. The PCR products were electrophoresed on 1.5\% agarose gels (Qiagen GmbH, Hilden, Germany), stained with $0.5 \mu \mathrm{g} / \mathrm{ml}$ ethidium bromide (Sigma-Aldrich), and images were captured using Gel Doc (Bio-Rad Laboratories, Inc., Milan, Italy). The densitometric analysis of the bands was determined using Image Lab software (version 2.2.4.0; MCM Design, Hillerød, Denmark) in order to compare the mRNA expression levels among samples, using GADPH as the standard gene. All quantification was performed in triplicate, and compared with DMSO $(0.1 \%)$ and rosiglitazone $(50 \mu \mathrm{M})$.

MitoTracker Red staining. The fluorescence signal of MitoTracker Red was used as an indicator of mitochondrial number. The differentiated cells grown on coverslips were incubated with media containing 5\% FBS and $200 \mathrm{nM}$ MitoTracker Red CMXRos (Molecular Probes; Thermo Fisher Scientific, Inc.) for $20 \mathrm{~min}$ in a $5 \% \mathrm{CO}_{2}$ incubator at $37^{\circ} \mathrm{C}$, followed by two washes in PBS. The cells were subsequently fixed with $4 \%$ paraformaldehyde (Bio Basic Canada, Inc., Markham, ON, Canada) for $15 \mathrm{~min}$, following which the cells were permeabilized with $0.5 \%$ Triton X-100 (Sigma-Aldrich) in PBS for $5 \mathrm{~min}$, and nuclei were stained with 4',6-diamidino-2-phenylindole (Roche Diagnostics, Indianapolis, IN, USA) at room temperature for an additional $10 \mathrm{~min}$. Immediately following a final wash with $0.1 \%$ Triton X-100 in PBS, images of the cells were captured under a Nikon microscope (ECLIPSE E600; Nikon Instruments, Melville, NY, USA).

Analysis of mitochondria membrane potential. The mitochondrial membrane potential was measured by means of 3,3'dihexyloxacarbocyanine iodide $\left(\mathrm{DiOC}_{6} 3\right)$ staining (Molecular Probes; Thermo Fisher Scientific, Inc.). The differentiated cells were harvested, washed three times in serum-free media and incubated in $200 \mathrm{nM} \mathrm{DiOC}_{6}$ (3)-containing media at $37^{\circ} \mathrm{C}$ for $20 \mathrm{~min}$. For each sample, at least 10,000 cells were analyzed for corresponding fluorescence on a flow cytometer (BD FACSCalibur ${ }^{\mathrm{TM}}$; BD Biosciences, Franklin Lakes, NJ, USA) using the FL1-H channel.

Computational analysis. Interruptin B was independently docked into the ligand-binding domain (LBD) of PPAR- $\alpha$ (PDB ID: $117 \mathrm{G})$ and PPAR- $\gamma$ (PDB ID: 4EMA). Ciglitazone, pioglitazone and rosiglitazone were used as positive controls for the PPAR- $\gamma$ ligands, according to standard procedure (18). Docking was performed using the AutoDock4.2 program suite $(19,20)$ from Scripps Research Institute (La Jolla, CA, USA). This program uses a Larmarckian genetic algorithm for the docking of flexible ligands into protein binding sites, in order to determine the full range of potential ligand conformers within a rigid protein target. The AutoDock run parameters used for all of the docking were a population size, of 150 and an increase in the maximum number of energy evaluations to $10,000,000$ per run. All other run parameters remained at their default settings. A total of 100 genetic algorithm runs were performed for each docking, and the final
Table I. Primers used for polymerase chain reaction amplification.

\begin{tabular}{ll} 
Primer & \multicolumn{1}{c}{ Sequence } \\
\hline PPAR- $\alpha \_F$ & 5'-CTGAGCCATGCAGAATTTAC-3' \\
PPAR- $\alpha \_R$ & 5'-TAACAGTTCCCTGAAGAGCA-3' \\
PPAR- $\gamma$ F & 5'-TGGAATTAGATGACAGCGACTTGG-3' \\
PPAR- $\gamma \_R$ & 5'-CTGGAGCAGCTTGGCAAACA-3' \\
C/EBP- $\beta$ _F & 5'-GTTCATGCAACGCCTGGTG-3' \\
C/EBP- $\beta$ _R & 5'-AAGCAGTCCGCCTCGTAGTAGAAG-3' \\
UCP-1_F & 5'-GTGTGCCCAACTGTGCAATG-3' \\
UCP-1_R & 5'-CCAGGATCCAAGTCGCAAGA-3' \\
CPT1B_F & 5'-AAACAGTGCCAGGCGGTC-3' \\
CPT1B_R & 5'-CGTCTGCCAACGCCTTG-3' \\
COX-2_F & 5'-CCCGCAGTACAGAAAGTATC-3' \\
COX-2_R & 5'-CCATAGAGTGCTTCCAACTC-3' \\
GLUT-1_F & 5'-ATCCCTGTTACCCAGAGAAT-3' \\
GLUT-1_R & 5'-TTCAGGCACATAACCTCTTT-3' \\
GLUT-4_F & 5'-GAATACCTTCTTCGCTGCTA-3' \\
GLUT-4_R & 5'-TGGATTTCTTGTCTCCTGTC-3' \\
GAPDH_F & 5'-CGAGATCCCTCCAAAATCAA-3' \\
GAPDH_R & 5'-TGTGGTCATGAGTCCTTCCA-3'
\end{tabular}

PPAR, peroxisome proliferator-activated receptor; C/EBP; CCAAT/ enhancer binding protein; UCP uncoupling protein; CPT1B, carnitine palmitoyltransferase 1B; COX; cyclooxygenase; GLUT; glucose transporter; GAPDH, glyceraldehyde-3-phosphate dehydrogenase; F, forward; R, reverse.

docked conformations were clustered using a tolerance of $2 \AA$ root mean square deviation (RMSD).

Transfection and luciferase assay. HepG2 cells [5x105; American Type Culture Collection (ATCC), Manassas, VA, USA] were seeded onto 12-well plates and transfected with lipofectamine 2000 (Invitrogen; Thermo Fisher Scientific, Inc.) and pGL3 Basic Vector (Promega Corporation) containing a PPAR response element (PPRE). In all cases, a $\beta$-galactosidase $(\beta$-gal) expression plasmid was transfected to control transfection efficiency. At $24 \mathrm{~h}$ post-transfection, the cells were treated with rosiglitazone $(10 \mu \mathrm{M})$ or interruptin B $(10 \mu \mathrm{M})$ for another $24 \mathrm{~h}$ at $37^{\circ} \mathrm{C}$. The cells were then washed twice with PBS and harvested using lysis buffer (Promega Corporation). Luciferase activity was determined using a Luciferase Assay system (Promega Corporation) and quantified using the Glomax 9101-002 system (Promega Corporation), according to the manufacturer's protocol. Luciferase activity was expressed as the fold induction, which was normalized by $\beta$-gal activity. Each condition was performed in triplicate.

PPAR ligand-binding activity assay. pcDNA3 vectors (Invitrogen; Thermo Fisher Scientific, Inc.) encoding HA-PPAR $\alpha$ and HA-PPAR $\gamma$ were transfected into HEK 293 cells $\left(2 \times 10^{6} / 100 \mathrm{~mm}\right.$; ATCC) for $24 \mathrm{~h}$ at $37^{\circ} \mathrm{C}$, and the cells were lysed with lysis buffer containing $50 \mathrm{mM}$ HEPES, $150 \mathrm{mM} \mathrm{NaCl}, 1 \mathrm{mM}$ EDTA, 10\% glycerol, Triton X-100, 
A<smiles>Cc1c(O)c(C(=O)/C=C/c2ccccc2)c2oc(=O)cc(-c3ccccc3)c2c1O</smiles>

Interruptin B

B

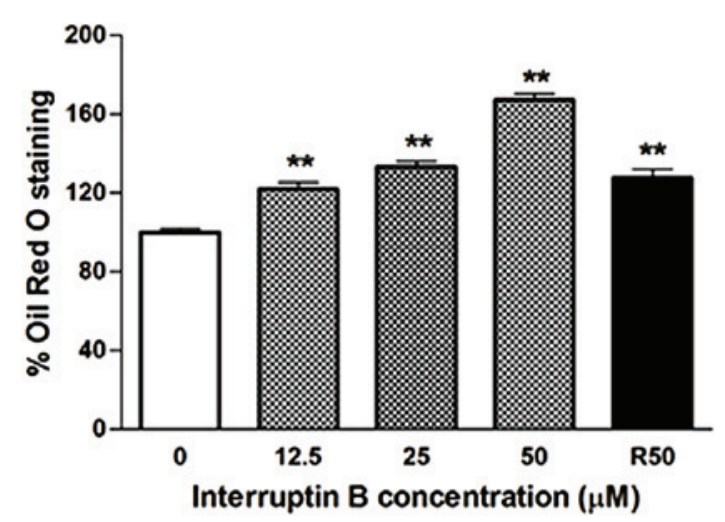

D

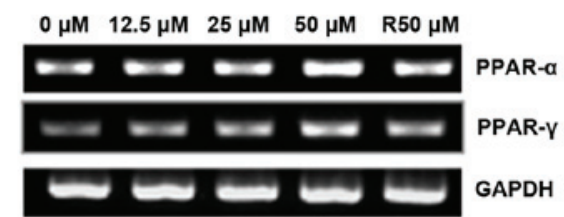

C
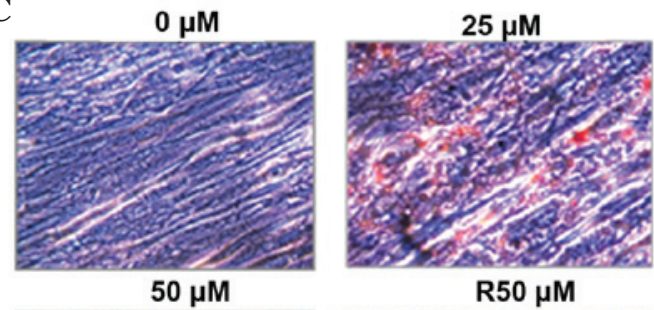

$\mathrm{R} 50 \mu \mathrm{M}$

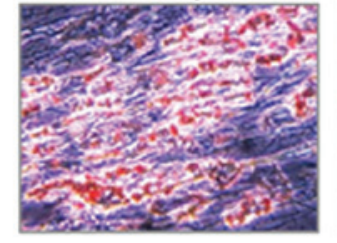

$\mathbf{E}$

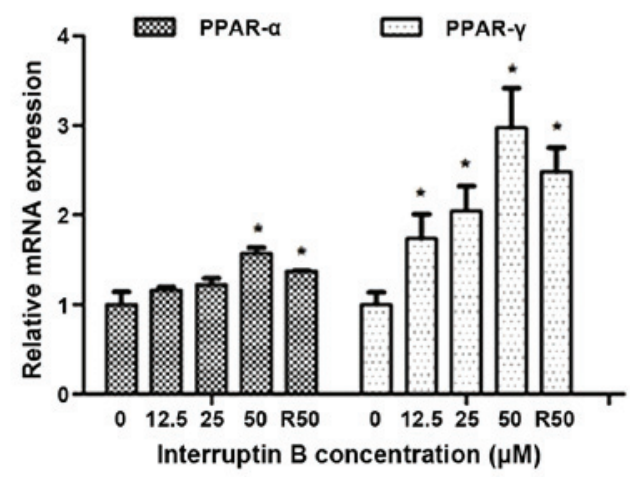

Figure 1. Chemical structure and adipocyte differentiation of interruptin B. (A) Chemical structure of interruptin B. ASCs were grown and differentiated in the absence and presence of interruptin B $(0,12.5,25$ or $50 \mu \mathrm{M})$. (B) Lipid accumulation was measured and (C) ASCs were stained with Oil Red O following 4 days of interruptin B treatment, demonstrating that interruptin B induced adipocyte differentiation of ASCs (magnification, $\mathrm{x} 40$ ). (D) mRNA expression levels of PPAR- $\alpha$ and PPAR- $\gamma$ were measured following 4 days of interruptin B treatment and (E) quantified. All values are presented as the mean \pm standard error of the mean $(n=3)$. ${ }^{*} \mathrm{P}<0.05$ and ${ }^{* *} \mathrm{P}<0.01$, compared with the untreated group. $\mathrm{R}$, rosiglitazone; PPAR, peroxisome proliferator-activated receptor; GAPDH, glyceraldehyde-3-phosphate dehydrogenase; ASCs, adipose-derived stem cells.

$12 \mathrm{mM} \beta$-glycerophosphate, $10 \mathrm{mM} \mathrm{NaF}, 1 \mathrm{mM} \mathrm{NaOV}_{3}$, $5 \mathrm{mg} / \mathrm{ml}$ aprotinin and $1 \mathrm{mM}$ PMSF (pH 7.6). Protein extracts were subsequently incubated with anti-HA agarose beads (Sigma-Aldrich) and washed with lysis buffer at $4^{\circ} \mathrm{C}$. Proteins were eluted using $0.1 \mathrm{M}$ glycine ( $\mathrm{pH} 2.5$; Sigma-Aldrich) and immediately neutralized with $1 \mathrm{M}$ Tris- $\mathrm{HCl}$ (Bio Basic Canada, Inc.). The proteins were then dialyzed against PBS containing $20 \%$ glycerol and stored at $-70^{\circ} \mathrm{C}$.

To analyze the interactions of interruptin $\mathrm{B}$ with binding affinities to myc-PPAR $\alpha$ and myc-PPAR $\gamma$, the surface plasmon resonance (SPR) technology of the SR7500DC system (Reichart Technologies, Buffalo, NY, USA) was used. The SPR experiment was performed according to the manufacturer's protocol. Protein quantitation was performed using a Bradford assay (Bio Rad Laboratories, Inc.), according to the manufacturer's instructions, with $0.6 \mu \mathrm{g}$ protein diluted in PBS. The proteins diluted in PBS were immobilized on a CMDH chip (Reichart Technologies), and interruptin B was loaded in a dose-dependent manner $(31.25,62.5,125,250$ and $500 \mu \mathrm{M})$ for the SPR assay. The equilibrium dissociation constant $\left(\mathrm{K}_{\mathrm{D}}\right)$ value was determined using the Scrubber2 program (Informer Technologies, Inc., Dallas, TX, USA).

Inhibition experiments. The roles of PPAR- $\alpha$ and PPAR- $\gamma$ were determined by measuring interruptin B-induced adipogenesis and glucose uptake in the presence or absence of PPAR- $\alpha$ and PPAR- $\gamma$ antagonists. The confluent ASCs were pretreated with 50 and $100 \mu \mathrm{M}$ GW6471 (PPAR- $\alpha$ inhibitor; Tocris Bioscience) or BADGE (PPAR- $\gamma$ inhibitor; Sigma-Aldrich) for $2 \mathrm{~h}$. Subsequently, the media were replaced with PDM-2, 

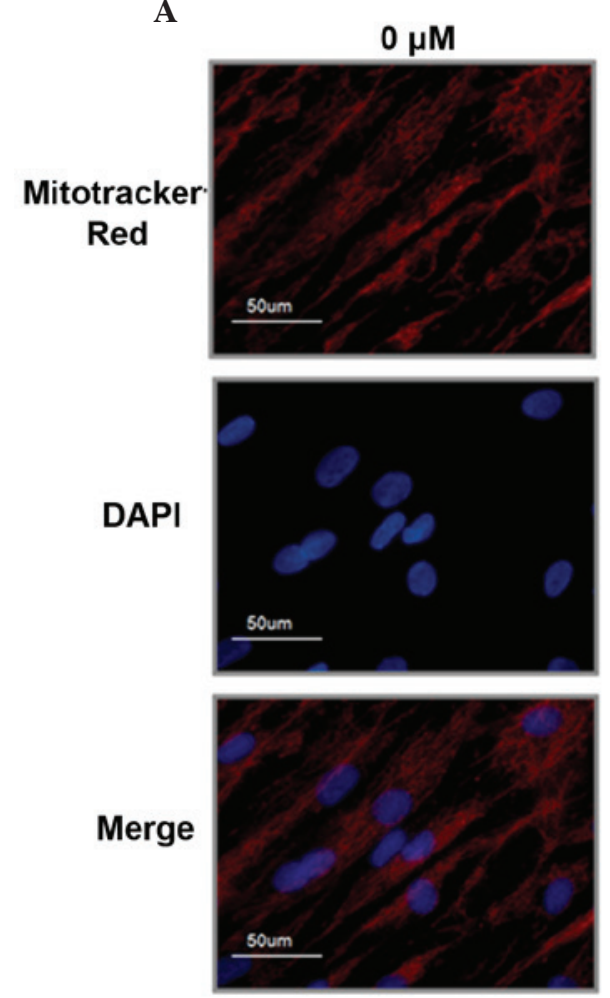

D
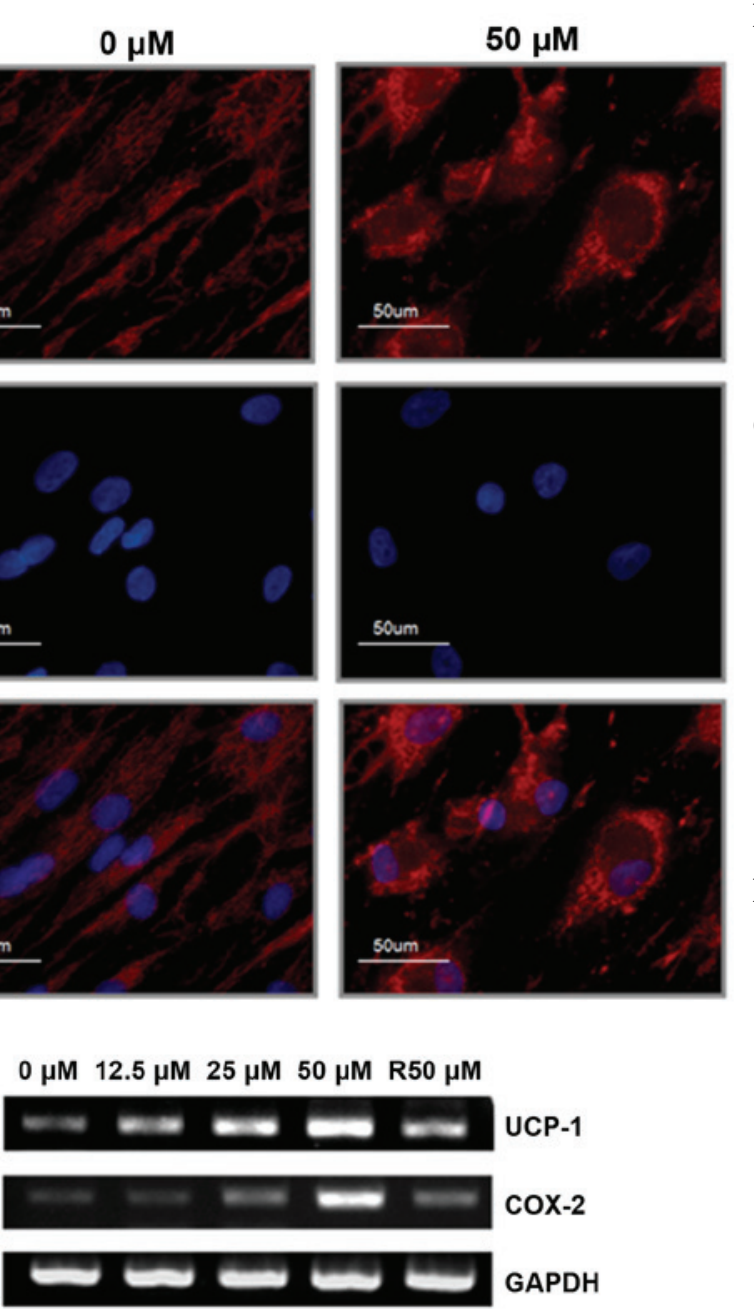

B

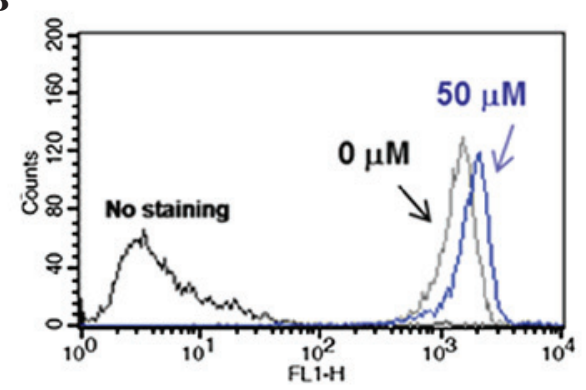

C

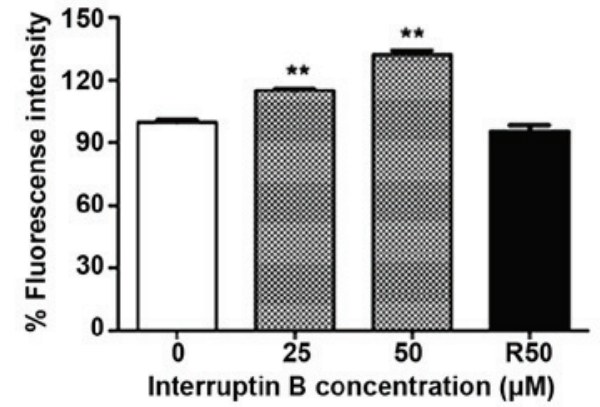

$\mathbf{E}$

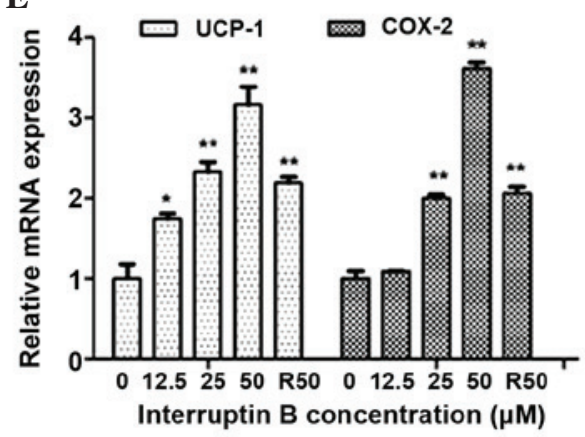

Figure 2. Effect of interruptin B on the brown adipocyte differentiation of ASCs. ASCs were grown and differentiated in the absence and presence of interruptin B $(0,12.5,25$ or $50 \mu \mathrm{M})$. (A) Signal density of MitoTracker Red following 4 days treatment with interruptin B $(50 \mu \mathrm{M})$. (B and C) Mitochondria membrane potential was measured by $\mathrm{DiOC}_{6}$ (3) staining following 4 days of treatment with interruptin B. (D) mRNA expression levels of UCP-1 and COX-2 were measured following 4 days treatment with interruptin $\mathrm{B}$ and (E) quantified. All values are presented as the mean \pm standard error of the mean $(\mathrm{n}=3)$. ${ }^{*} \mathrm{P}<0.05$ and ${ }^{* *} \mathrm{P}<0.01$, compared with the untreated group. R, rosiglitazone; UCP-1, uncoupling protein-1; COX, cyclooxygenase; GAPDH, glyceraldehyde-3-phosphate dehydrogenase; DAPI, 4',6-diamidino-2-phenylindole; ASCs, adipose-derived stem cells.

comprised of specific inhibitors and $50 \mu \mathrm{M}$ interruptin B. Lipid accumulation and glucose uptake were examined following culture for 4 days at $37^{\circ} \mathrm{C}$.

Statistical analysis. Data are presented as the mean \pm standard error of the mean. Results were analyzed and presented using GraphPad Prism ${ }^{\circledR}$ version 6.00 (GraphPad Software, San Diego, CA, USA). Comparisons between two groups were performed using Student's t-test and $\mathrm{P}<0.05$ was considered to indicate a statistically significant difference.

\section{Results}

Interruptin $B$ enhances adipocyte differentiation. To investigate the effect of interruptin B on adipocyte differentiation, confluent ASCs were treated with various concentrations (12.5-50 $\mu \mathrm{M})$ of interruptin B for 4 days, following which the ASCs were fixed and stained with Oil Red O in order to detect accumulated neutral lipid. Microscopic examination of the stained dishes demonstrated that interruptin B increased the lipid accumulation in a dose-dependent manner, with an increase of $121.9-167.2 \%$, whereas the increase in the cells treated with rosiglitazone at $50 \mu \mathrm{M}$ was only $127.7 \%$ (Fig. 1B and $\mathrm{C}$ ).

To further characterize the molecular changes induced by interruptin B during adipocyte differentiation, the present study examined the mRNA expression levels of PPAR- $\gamma$ and PPAR- $\alpha$ during ASC differentiation (Fig. 1D and E). The mRNA expression levels of PPAR- $\alpha$ and PPAR- $\gamma$ were significantly enhanced during interruptin B-induced ASC differentiation, in a concentration-dependent manner. Rosiglitazone at $50 \mu \mathrm{M}$ also produced a positive effect on the adipogenesis markers. The interruptin B-induced mRNA expression of PPAR- $\gamma$ was two-fold higher, compared with that of PPAR- $\alpha$.

Interruptin B facilitates brown adipocyte differentiation. There is increasing evidence to suggest that mitochondria are important during brown adipocyte differentiation, and that the brown color is a result of the high concentrations of mitochondria, which are not found in white adipocytes $(21,22)$. Therefore, 
A

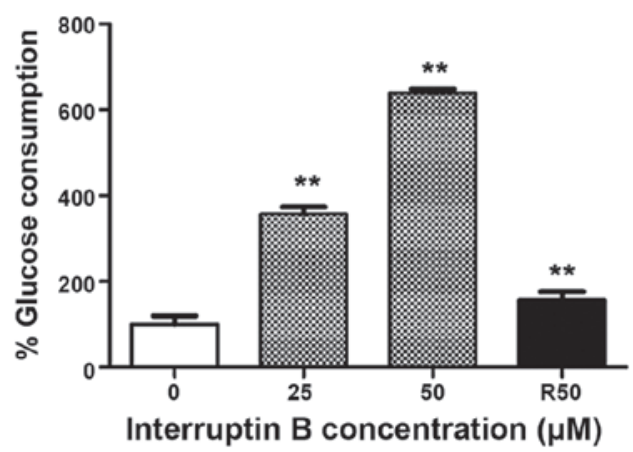

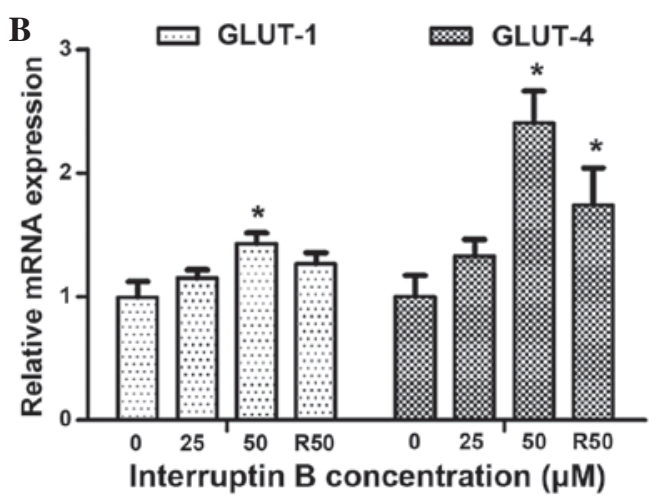

C

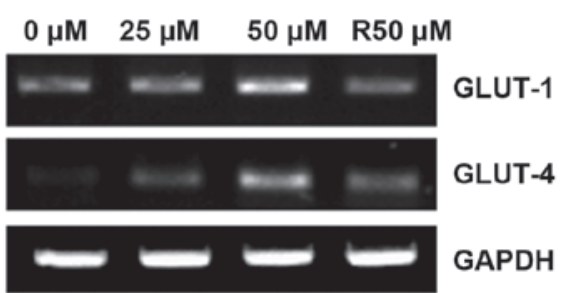

Figure 3. Effect of interruptin B on glucose uptake during adipocyte differentiation of ASCs. ASCs were grown and differentiated in the absence and presence of interruptin B $(0,25$ or $50 \mu \mathrm{M})$. (A) Glucose consumption was measured following 4 days treatment with interruptin B. (B) mRNA expression levels of GLUT-1 and GLUT-4 were measured following 4 days of interruptin B treatment and (C) quantified. All values are presented as the mean \pm tandard error of the mean $(n=3) . ~{ }^{*} \mathrm{P}<0.05$ and ${ }^{* *} \mathrm{P}<0.01$, compared with the untreated group. R, rosiglitazone; GLUT; glucose transporter; GAPDH, glyceraldehyde-3-phosphate dehydrogenase; ASCs, adipose-derived stem cells.

A

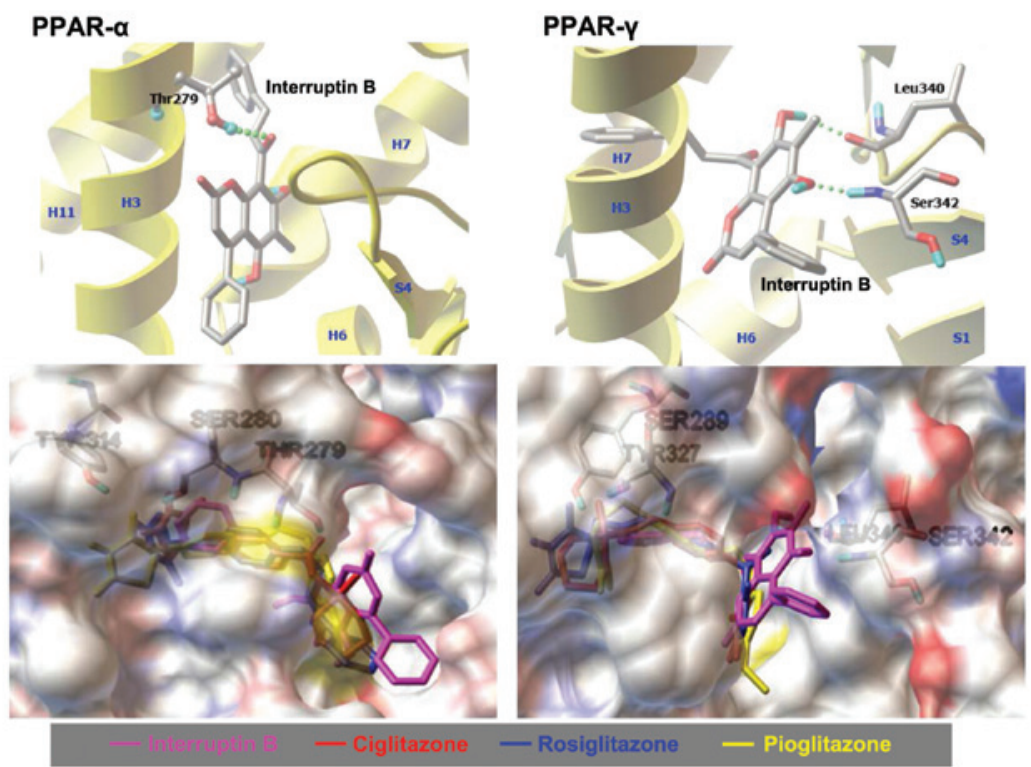

B

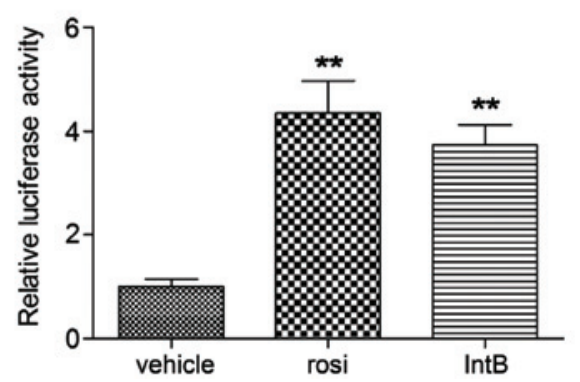

Figure 4. Predicted binding of interruptin B in the ligand-binding domain of PPAR- $\alpha$ (1I7G) and PPAR- $\gamma$ (4EMA) by molecular docking in silico, and the effect of interruptin B on PPAR activation in HepG2 cells. (A) Structural superposition of interruptin B, compared with the PPAR- $\gamma$ ligand. Hydrogen bond interactions are shown with a green dotted line. Secondary structure elements are shown in yellow. The structures of interruptin B, ciglitazone, rosiglitazone, and pioglitazone are shown in pink, red, blue and yellow, respectively. HepG2 cells were transfected with PPAR response element-containing reporter plasmids. (B) Luciferase and $\beta$-gal activities were assessed following $24 \mathrm{~h}$ treatment with interruptin B or rosiglitazone $(10 \mu \mathrm{M})$, All values are presented as the mean \pm standard error of the mean $(n=3) .{ }^{* *} \mathrm{P}<0.01$, compared with the untreated group. PPAR, peroxisome proliferator-activated receptor; Int $\mathrm{B}$, interruptin $\mathrm{B}$; rosi, rosiglitazone. 
Table II. In silico docking results of interruptin B, compared with the PPAR- $\gamma$ ligands to PPAR- $\alpha$ and PPAR- $\gamma$, using the AutoDock4.2 program.

\begin{tabular}{|c|c|c|c|c|c|c|}
\hline \multirow[b]{2}{*}{ Compound } & \multicolumn{3}{|c|}{ PPAR- $\alpha$ (PDB: 1I7G) } & \multicolumn{3}{|c|}{ PPAR- $\gamma$ (PDB: 4EMA) } \\
\hline & $E_{\text {bind }}{ }^{\mathrm{a}}(\mathrm{kcal} / \mathrm{mol})$ & $\operatorname{Member}^{\mathrm{b}}(\%)$ & Interacting residue $^{c}$ & $E_{\text {bind }}{ }^{\mathrm{a}}(\mathrm{kcal} / \mathrm{mol})$ & $\operatorname{Member}^{\mathrm{b}}(\%)$ & Interacting residue $^{c}$ \\
\hline Interruptin B & -9.29 & 59 & Thr279 & -9.36 & 43 & Leu340, Ser342 \\
\hline Ciglitazone & -8.5 & 24 & Thr279, Ser280 & -8.8 & 41 & - \\
\hline Pioglitazone & -9.44 & 28 & Thr279, Ser280, Tyr314 & -8.5 & 25 & Tyr327 \\
\hline Rosiglitazone & -7.83 & 21 & Thr279, Ser280 & -8.76 & 82 & Ser289 \\
\hline
\end{tabular}

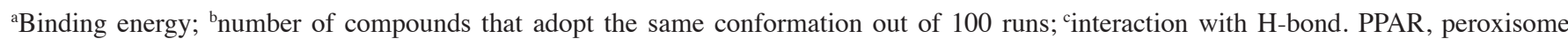
proliferator-activated receptor.

A

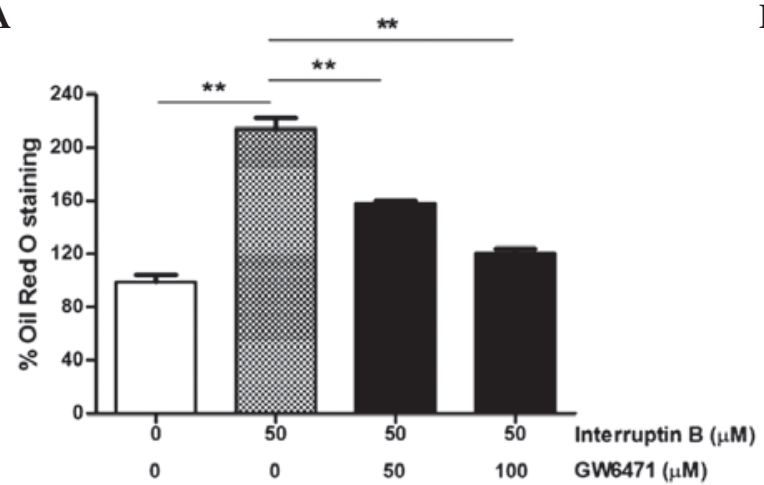

C

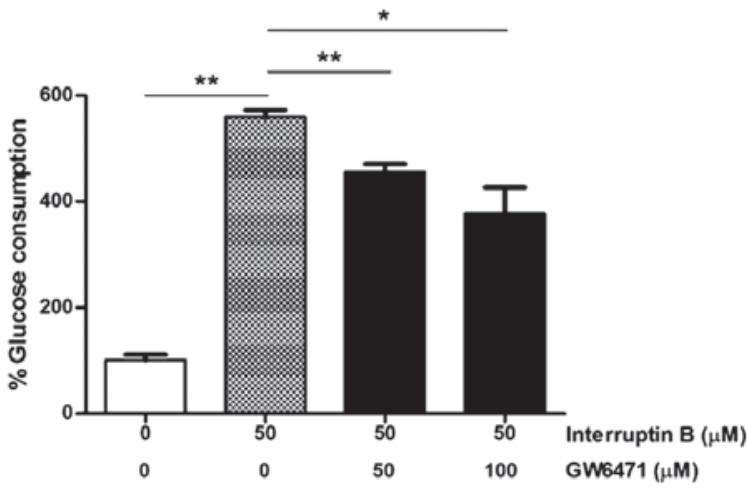

B

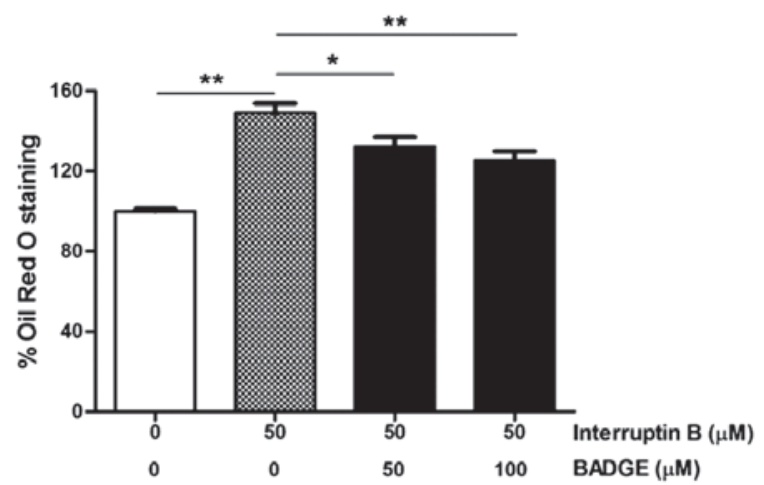

D

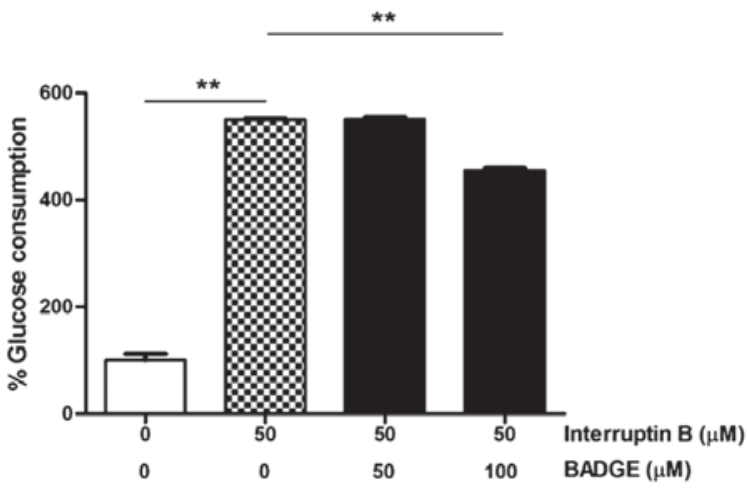

Figure 5. Reversal of adipocyte differentiation and glucose consumption by PPAR- $\alpha$ (GW6471) and PPAR- $\gamma$ (BADGE) antagonists. Confluent adipose-derived stem cells were pretreated with GW6471 or BADGE $(0,50$ or $100 \mu \mathrm{M})$ for $2 \mathrm{~h}$ and subsequently differentiated by interruptin B (50 $\mu \mathrm{M})$ in the absence and presence of GW6471 or BADGE. (A and B) Lipid accumulation and (C and D) glucose consumption were measured following 4 days of interruptin B treatment. All values are presented as the mean \pm standard error of the mean $(n=3)$. ${ }^{*}<0.05$ and ${ }^{* *} \mathrm{P}<0.01$, compared with the untreated group. PPAR, peroxisome proliferator-activated receptor.

the present study examined whether interruptin B had any effect on mitochondrial number or membrane potential during differentiation. Fluorescence microscopy using a mitochondrial-specific dye, MitoTracker Red, showed that interruptin $\mathrm{B}$ increased mitochondrial number in the ASCs, compared with the control. In addition, interruptin B treatment caused an enlargement in cell size during ASC differentiation (Fig. 2A). The mitochondrial membrane potential was examined using $\mathrm{DiOC}_{6}$ (3) staining. The fluorescence intensities of $\mathrm{DiOC}_{6}(3)$ were significantly increased by 115.1 and $132.3 \%$ in the 25 and $50 \mu \mathrm{M}$ interruptin B-treated cells, respectively, compared with the control (Fig. 2B). However, the mitochondrial membrane potential was not induced by $50 \mu \mathrm{M}$ rosiglitazone treatment (Fig. 2C).

Exposure to interruptin $\mathrm{B}(12.5-50 \mu \mathrm{M})$ induced the mRNA expression levels of the brown adipocyte markers, uncoupling protein-1 (UCP-1) and COX-2, compared with the control (1.7- and 3.6-fold, respectively), and $50 \mu \mathrm{M}$ rosiglitazone increased the expression levels 2.2- and 2.0-fold, respectively (Fig. 2D and E).

Interruptin B increases glucose consumption. As PPAR- $\gamma$ is the molecular target for antidiabetic drugs, which improve insulin sensitivity and glucose tolerance, the present study examined 
Table III. $\mathrm{K}_{\mathrm{D}}$ values of interruptin B on PPAR- $\alpha$ and PPAR- $\gamma$, determined using a surface plasmon resonance assay.

\begin{tabular}{lc}
\hline Protein & $\mathrm{K}_{\mathrm{D}}(\mu \mathrm{M})$ \\
\hline PPAR- $\alpha$ & $5.32 \pm 0.77$ \\
PPAR- $\gamma$ & $0.10 \pm 0.00$ \\
\hline
\end{tabular}

$\mathrm{K}_{\mathrm{D}}$ values are presented as the mean \pm standard error of the mean; PPAR, peroxisome proliferator-activated receptor; $\mathrm{K}_{\mathrm{D}}$, equilibrium dissociation constant.

whether interruptin B treatment affects glucose metabolism, compared with rosiglitazone. As shown in Fig. 3A, glucose consumption was significantly increased (357-640\%) in the differentiated ASCs treated with 25 and $50 \mu \mathrm{m}$ interruptin B, which was 2.3-4.1 times higher than the glucose consumption measured following treatment with $50 \mu \mathrm{m}$ rosiglitazone $(157 \%)$.

To understand the mechanism underlying the effects of interruptin B on glucose consumption, the mRNA expression levels of a facilitated glucose transporter system were determined. As shown in Fig. 3B and C, the mRNA expression levels of GLUT-1 and GLUT-4 were significantly upregulated in the ASCs exposed to $50 \mu \mathrm{M}$ of interruptin B, compared with the untreated control cells.

Interruptin B is a dual PPAR- $\alpha$ and PPAR- $\gamma$ ligand.Molecular docking of interruptin $B$ was performed with AutoDock v4.2 using the X-ray crystal structures PDB ID: 1 I7G and 4EMA as templates for PPAR- $\alpha$ and PPAR- $\gamma$, respectively. Each compound was docked 100 times, and those conformations exhibiting similar orientation (RMSD $<2 \AA$ ) were clustered. Table II lists the estimated protein-ligand binding affinities of interruptin B, compared with ciglitazone, pioglitazone and rosiglitazone (standard PPAR- $\gamma$ ligands). A more negative value of binding energy and a higher percentage of members in a cluster correspond to a higher predicted binding affinity (23). Interruptin $\mathrm{B}$ was predicted to be a robust PPAR ligand, having achieved a relatively favorable docking score and relying on the same receptor pockets as observed for ciglitazone, pioglitazone and rosiglitazone. The hydrogen-bond contacts of interruptin B interacted with Thr279 in PPAR- $\alpha$, and Leu340 and Ser342 in PPAR- $\gamma$ (Fig. 4A). In addition, there was increased binding energy to PPAR- $\alpha$ and PPAR- $\gamma$ than for rosiglitazone, which was used as the positive control.

To confirm that interruptin B activated PPARs, the present study assessed its ability to directly activate the PPAR ligand-binding domain using a chimeric PPRE fusion protein on a pGL3-dependent luciferase reporter. As shown in Fig. 4B, $10 \mu \mathrm{M}$ interruptin B considerably increased PPAR- $\alpha$ and PPAR- $\gamma$ transcriptional activity by 3.7 -fold, with rosiglitazone causing a 4.4-fold increase. In addition, the binding activities of interruptin B to purified PPAR- $\alpha$ and PPAR- $\gamma$ were determined using an SPR assay (Table III). The results confirmed that interruptin B bound to PPAR- $\alpha$ and PPAR- $\gamma$, with $K_{D}$ values of 5.32 and $0.10 \mu \mathrm{M}$, respectively. These data indicated that interruptin B acted as a dual PPAR ligand, with superior PPAR- $\gamma$ to PPAR- $\alpha$ binding.

Functional inhibition by PPAR antagonists. To support the activity of interruptin B as a dual PPAR- $\alpha$ and PPAR- $\gamma$ ligand, the present study performed inhibition experiments in ASC development against specific PPAR- $\alpha$ and $-\gamma$ inhibitors. Compared with the control, ASCs treated with $50 \mu \mathrm{m}$ interruptin B exhibited increased lipid accumulation, as determined by Oil Red O staining, which was significantly attenuated by treatment with 50 and $100 \mu \mathrm{M}$ GW6471 and BADGE (PPAR- $\alpha$ and PPAR- $\gamma$ antagonists, respectively; Fig. 5A and B). Similarly, the increased glucose consumption, which was induced by $50 \mu \mathrm{M}$ interruptin B was reduced considerably by co-treatment with 50 or $100 \mu \mathrm{M} \mathrm{GW6471, \text {and }}$ $100 \mu \mathrm{M}$ BADGE (Fig. 5C and D). The results suggested that the interruptin B-induced adipocyte differentiation and glucose consumption were eradicatd in a dose-dependent manner by GW6471 and BADGE. Taken together, these observations supported that the induction of adipocyte differentiation and glucose uptake by interruptin B was dependent on PPAR- $\alpha$ and PPAR- $\gamma$ activation.

\section{Discussion}

In a preliminary investigation in the present study, interruptin B not only induced ASC proliferation, but also markedly enhanced cell size (data not shown). Therefore, whether interruptin B was involved in the adipogenesis of ASCs to produce brown adipocyte tissue was subsequently investigated. The results of the present study demonstrated for the first time, to the best of our knowledge, that interruptin $\mathrm{B}$ induced brown adipocyte differentiation and increased glucose uptake in ASCs. In addition, interruptin B showed increased adipogenic differentiation potential and glucose uptake, compared with rosiglitazone. Although the effects of natural compounds, including daidzein, equol, magnolol and arteplilin $\mathrm{C}$, for the induction of adipocyte differentiation and glucose uptake have been demonstrated, they all act as ligands of PPAR- $\gamma$ and originate from cultivated plants (24-26). To the best of our knowledge, the present study is the first report to show the stimulation of adipocyte differentiation and glucose uptake by interruptin B from the wild plant, $C$. terminans, mediated by the dual effects of PPAR- $\alpha$ and PPAR- $\gamma$ binding.

To predict the specific target ASC receptors that respond to interruptin B in the present study, computational analysis was performed using the AutoDock4.2 program. In comparison with standard PPAR- $\gamma$ sensitizers, ciglitazone, pioglitazone, and rosiglitazone, molecular docking predicted interruptin B as a dual PPAR- $\alpha$ and $-\gamma$ ligand. In addition, a luciferase reporter assay and SPR technology demonstrated that interruptin B acted as a dual PPAR- $\alpha$ and PPAR- $\gamma$ agonist. In the inhibition experiments, interruptin B-induced adipocyte differentiation and glucose uptake were significantly inhibited, in a dose-dependent manner, by co-treatment with the PPAR- $\alpha$ antagonist GW6471 or the PPAR-g antagonist BADGE. These data confirmed that adipocyte differentiation and glucose consumption were enhanced by interruptin B treatment through the PPAR- $\alpha$ and PPAR- $\gamma$ dependent pathway. 
TZDs have been investigated as potential antidiabetic drugs. Rosiglitazone is a selective PPAR- $\gamma$ agonist, while pioglitazone exerts PPAR- $\alpha$ and PPAR- $\gamma$ agonistic activity, which may cause different metabolic effects. However, rosiglitazone has been associated with an enhanced risk of myocardial infarction, which led to the withdrawal of this drug from the market in 2010 (27). By contrast, the beneficial vascular effects of pioglitazone, owing to its dual PPAR- $\alpha$ and PPAR- $\gamma$ binding, have been established. Therefore, investigations are focusing on other dual PPAR- $\alpha$ and PPAR- $\gamma$ agonists to improve not only glycemic control, but also lipid levels, potentially reducing vascular risk (28). The combined PPAR- $\alpha$ and PPAR- $\gamma$ stimulation effects of interruptin B may, therefore, offer an attractive option for developing antidiabetic or anti-obesity drugs with reduced cardiovascular risk.

In conclusion, the present study demonstrated that interruptin $\mathrm{B}$, an ingredient of $C$. terminans, induced brown adipocyte differentiation. In addition, the downstream responses to PPAR- $\alpha$ and PPAR- $\gamma$ activation included increases in the mRNA expression levels of GLUT-1 and GLUT-4, resulting in facilitated glucose uptake. Although its corresponding effects in vivo remain to be elucidated, these data indicate that interruption $\mathrm{B}$ is a potential natural insulin sensitizer from ferns. These novel findings support the benefits of C.terminans consumption, and may assist in the progression between epidemiological observations and clinical studies on the antidiabetic or anti-obesity benefits of fern plants.

\section{Acknowledgements}

The authors would like to thank Dr Brian Hodgson of PSU for assistance with English.

\section{References}

1. Hauptman J, Lucas C, Boldrin MN, Collins H and Segal KR: Orlistat in the long-term treatment of obesity in primary care settings. Arch Fam Med 9: 160-167, 2000.

2. Haslam DW and James WP: Obesity. Lancet 366: 1197-1209, 2005.

3. Halford JC: Pharmacotherapy for obesity. Appetite 46: 6-10, 2006.

4. Filippatos TD, Derdemezis CS, Gazi IF, Nakou ES, Mikhailidis DP and Elisaf MS: Orlistat-associated adverse effects and drug interactions: A critical review. Drug Saf 31: 53-65, 2008.

5. Yao X, Shan S, Zhang Y and Ying H: Recent progress in the study of brown adipose tissue. Cell Biosci 1: 35, 2011.

6. Amos AF, McCarty DJ and Zimmet P: The rising global burden of diabetes and its complications: Estimates and projections to the year 2010. Diabet Med 14 (Suppl 5): S1-S85, 1997.

7. Takada I and Makishima M: PPAR $\gamma$ ligands and their therapeutic applications: a patent review (2008 - 2014). Expert Opin Ther Pat 25: 175-191, 2015.

8. Nathan DM: Diabetes: Advances in diagnosis and treatment JAMA 314: 1052-1062, 2015.
9. Fujimura T, Kimura C, Oe T, Takata Y, Sakuma H, Aramori I and Mutoh S: A selective peroxisome proliferator-activated receptor gamma modulator with distinct fat cell regulation properties. J Pharmacol Exp Ther 318: 863-871, 2006.

10. Teboul L, Gaillard D, Staccini L, Inadera H, Amri EZ and Grimaldi PA: Thiazolidinediones and fatty acids convert myogenic cells into adipose-like cells. J Biol Chem 270: 28183-28187, 1995.

11. Patel C, Wyne KL and McGuire DK: Thiazolidinediones, peripheral oedema and congestive cardiac failure: What is the evidence? Diab Vasc Dis Res 2: 61-66, 2005.

12. Semple RK, Chatterjee VK and O'Rahilly S: PPAR gamma and human metabolic disease. J Clin Invest 116: 581-586, 2006.

13. Diamond GA, Bax L and Kaul S: Uncertain effects of rosiglitazone on the risk for myocardial infarction and cardiovascular dealth. Ann Intern Med 147: 578-581, 2007.

14. Medina-Gómez G: Mitochondria and endocrine function of adipose tissue. Best Pract Res Clin Endocrinol Metab 26: 791-804, 2012.

15. Kumboonruang N: Fern diversity at Silaphet waterfall, Pua district, Nan province. (unpublished Master's Project, M.Ed.). Graduate School, Srinakharinwirot University, Bangkok, Thailand, 2009.

16. Kaewsuwan S, Yuenyongsawad S, Plubrukarn A, Kaewchoothong A, Raksawong A, Puttarak P and Apirug C: Biological activities of interruptins A and B from Cyclosorus terminans. Songklanakarin J Sci Technol (In press).

17. Kim WS, Park BS, Park SH, Kim HK and Sung JH: Antiwrinkle effect of adipose-derived stem cell: Activation of dermal fibroblast by secretory factors. J Dermatol Sci 53: 96-102, 2009.

18. Sherman W, Day T, Jacobson MP, Friesner RA and Farid R: Novel procedure for modeling ligand/receptor induced fit effects. J Med Chem 49: 534-553, 2006.

19. Kumari V and Li C: Comparative docking assessment of glucokinase interactions with its allosteric activators. Curr Chem Genomics 2: 76-89, 2008.

20. Morris GM, Huey R, Lindstrom W, Sanner MF, Belew RK, Goodsell DS and Olson AJ: AutoDock4 and AutoDockTools4: Automated docking with selective receptor flexibility. J Comput Chem 30: 2785-2791, 2009

21. Cannon B and Nedergaard J: Brown adipose tissue: Function and physiological significance. Physiol Rev 84: 277-359, 2004.

22. Rehman J: Empowering self-renewal and differentiation: The role of mitochondria in stem cells. J Mol Med (Berl) 88: 981-986, 2010.

23. Gallicchio E, Lapelosa M and Levy RM: The binding energy distribution analysis method (BEDAM) for the estimation of protein-ligand binding affinities. J Chem Theory Comput 6: 2961-2977, 2010

24. Cho KW, Lee OH, Banz WJ, Moustaid-Moussa N, Shay NF and Kim YC: Daidzein and the daidzein metabolite, equol, enhance adipocyte differentiation and PPARgamma transcriptional activity. J Nutr Biochem 21: 841-847, 2010.

25. Choi SS, Cha BY, Lee YS, Yonezawa T, Teruya T, Nagai K and Woo JT: Magnolol enhances adipocyte differentiation and glucose uptake in 3T3-L1 cells. Life Sci 84: 908-914, 2009.

26. Choi SS, Cha BY, Iida K, Lee YS, Yonezawa T, Teruya T, Nagai K and Woo JT: Artepillin C, as a PPAR $\gamma$ ligand, enhances adipocyte differentiation and glucose uptake in 3T3-L1 cells. Biochem Pharmacol 81: 925-933, 2011.

27. Nissen SE and Wolski K: Rosiglitazone revisted: An updated meta-analysis of risk for myocardial infarction and cardiovascular mortality. Arch Intern Med 170: 1191-1201, 2010.

28. Henry RR, Lincoff AM, Mudaliar S, Rabbia M, Chognot C and Herz M: Effect of the dual peroxisome proliferator-activated receptor-alpha/gamma agonist aleglitazar on risk of cardiovascular disease in patients with type 2 diabetes (SYNCHRONY): A phase II, randomised, dose-ranging study. Lancet 374: 126-135, 2009. 